analysis one patient in the black group had flare (14.2\%) five in the intravenous administration had severe flares(8.1\%) and were discontinued.During the evaluation ten had flares that were adjusted with steroids (eight articular or skin reactivation) and two with renal disease. On the five severe flares two required hospitalization. The mean time duration to achieve LLDAS was six months. Twenty seven achieved steroid free status and remained two patients on $2.5 \mathrm{mg}$ and seventeen stable on daily $5.0 \mathrm{mg}$ of prednisone.

Conclusions Using the LLDAS criteria response, it was possible to show that the majority of patients with active SLE on all three groups studied intravenous, subcutaneous and black race receiving belimumab for prolonged periods go into remission steroid free or in low disease activity in association with the correspondent immunosuppressive treatment.

Funding Source(s): Funding source Institutional and GSK.

\section{CLINICAL CHARACTERISTICS AND REMISSION OF PATIENTS WITH SYSTEMIC LUPUS ERYTHEMATOSUS IN CHINA: RESULTS FROM SLE TREATMENT AND RESEARCH GROUP (CSTAR) REGISTRY WITH A REAL- TIME COLLECTING SYSTEM}

${ }^{1}$ Ziqian Wang ${ }^{*},{ }^{2}$ Mengtao Li, ${ }^{2}$ Jiuliang Zhao, ${ }^{2}$ Qian Wang, ${ }^{2}$ Xiaofeng Zeng. ${ }^{1}$ Peking Union Medical College Hospital; ${ }^{2}$ Department of Rheumatology, Peking Union Medical College Hospital, Peking Union Medical College and Chinese Academy of Medical Science, Key Laboratory of Rheumatology and Clinical Immunology, Ministry of Education, Beijing, China

10.1136/lupus-2019-Ism.220

Background To develop a new system to study the real-world clinical characteristics and remission of systemic lupus erythematosus based on the first and largest registry cohort in China.

Methods Based on previous experience of CSTAR registry from 2009, a new online platform through mobile application program (APP)was designed to continuously collect realtime data during clinical practice with predesigned case report form. Baseline and follow-up datawere directly collected by physician at clinic, uploaded via our APP and integrated into database for analysis immediately, including demography, clinical manifestations, disease activity (SLEDAI$2 \mathrm{~K}$ ), organ damage (SLICC Damage Index), lab results, imaging and medications. Biological samples were preserved for future study. Along with data collection, data cleaning and validation were managed by a professional backstage statistician to ensure quality.

Results A total of 13,699 SLE patients from 236 different centers have been registered up to December 2018. The male to female rate was $1: 12(1076 / 12,623)$. The average age at onset, at diagnosis and at enrollment were $30.7 \mathrm{y}$, $31.7 \mathrm{y}$ and $35.5 \mathrm{y}$ respectively. The top three involved organ systems at entry were lupus nephritis (36\%), hematologic involvement $(35.8 \%)$ and neuropsychiatric disorder $(5.7 \%)$. In addition, 637 patients (4.6\%) were found to be diagnosed with pulmonary arterial hypertension, and 166 patients $(1.2 \%)$ had interstitial lung disease at baseline. The rate of clinical remission and complete remission at latest follow-up were $3.2 \%$ and $2.8 \%$.

Conclusions CSTAR firstly built a direct online reporting and collecting system, which can continuously register and follow SLE patients all around China, giving us an opportunity to study the real-world conditions and provide the possibility to apply Treat-To-Target strategy into daily practice for Chinese SLE patients

Funding Source(s): Chinese National Key Research R and D Program (2017YFC0907601, 2017YFC0907602, 2017YFC0907603), the Chinese National High Technology Research and Development Program, Ministry of Science and Technology (2012AA02A513) and the Chinese National Key Technology R and D Program, Ministry of Science and Technology (2008BAI59B02).

\section{META-ANALYSIS EXAMINING THE CLINICAL SIGNIFICANCE OF MONITORING OF HYDROXYCHLOROQUINE LEVELS IN SLE}

${ }^{1}$ Shivani Garg*, ${ }^{2}$ Rachna Unnithan, ${ }^{3}$ Karen Hansen, ${ }^{4}$ Nathalie Costedoat-Chalumeau, ${ }^{5}$ Christie Bartels. ${ }^{1}$ UW- Madison; ${ }^{2}$ University of Wisconsin- Madison; ${ }^{3}$ University of Wisconsin- Madison, Department of Medicine-Rheumatology; ${ }^{4}$ Unité fonctionnelle de Médecine interne et centre de référence maladies rares; ${ }^{5}$ University of Wisconsin School of Medicine and Public Health

\subsection{6/lupus-2019-Ism.221}

Background Despite the pivotal role hydroxychloroquine (HCQ) plays in treating SLE, less than 50\% of patients take HCQ as prescribed. Non-adherence versus lack of effect to HCQ are difficult to distinguish, underscoring the importance of measuring HCQ blood levels to assess adherence. Despite this, information and consensus on the clinical impact of incorporating routine testing of HCQ blood levels is lacking. Therefore, we systematically reviewed publications examining the correlation between 1) HCQ levels and adherence, and 2) HCQ levels and SLEDAI scores, in SLE patients. We hypothesized that low HCQ levels would correlate with non-adherence and higher SLEDAI scores.

Methods A comprehensive search was performed using $\mathrm{MeSH}$ heading and keywords in Medline, Embase, CINHL and Web of Science databases. We selected observational and interventional studies that measured HCQ levels and assessed adherence and/or SLEDAI scores in adults with SLE. Newcastle Ottawa Scale and Cochrane Collaboration Risk Assessment tools were used to rate the quality of observational and intervention studies, respectively. We used Forest plots to compare pooled estimates (95\% CI) of correlations between HCQ levels and patient or physician reported nonadherence and SLEDAI scores. Heterogeneity was assessed using I2.

Results From 306 manually reviewed studies, four studies analyzing correlation between HCQ levels and adherence, and five studies examining the correlation between SLEDAI and HCQ blood levels, met inclusion criteria. The odds of nonadherence measured by physician or reported by the patient was 3 times higher in patients with below threshold HCQ levels, compared to those with higher HCQ levels (OR 2.95, 95\% CI 1.63, 5.35, p<0.001, I2 49\%) (figure 1, panel A). The mean SLEDAI score was 3.33 points higher in groups with HCQ levels below threshold, but this trend was not statistically significant $(3.33,95 \%$ CI $-0.60,7.26, p=0.097$, I2 99\%) (figure 1, panel B). Risk of bias assessment revealed three poor quality studies which were excluded in sensitivity analysis which did not change results. Limitations of our analysis include study heterogeneity and lack of consensus on HCQ level interpretations.

Conclusions We found good correlation between HCQ levels and non-adherence. Low HCQ levels showed higher mean 
Panel A. Forest Plot of correlation of measured HCQ blood levels with assessed adherence

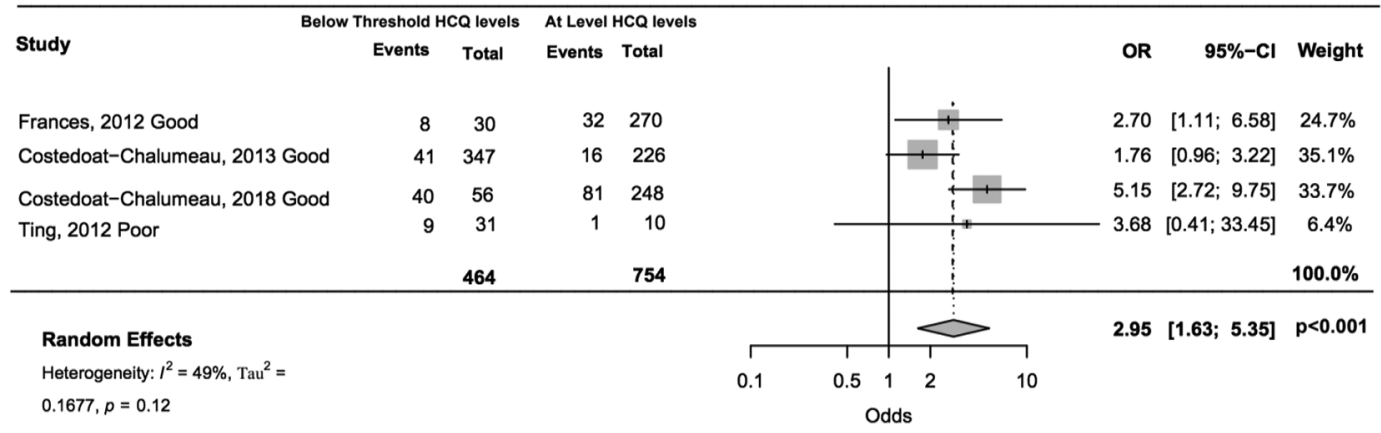

Panel B. Forest Plot of correlation of measured HCQ levels with SLEDAI scores

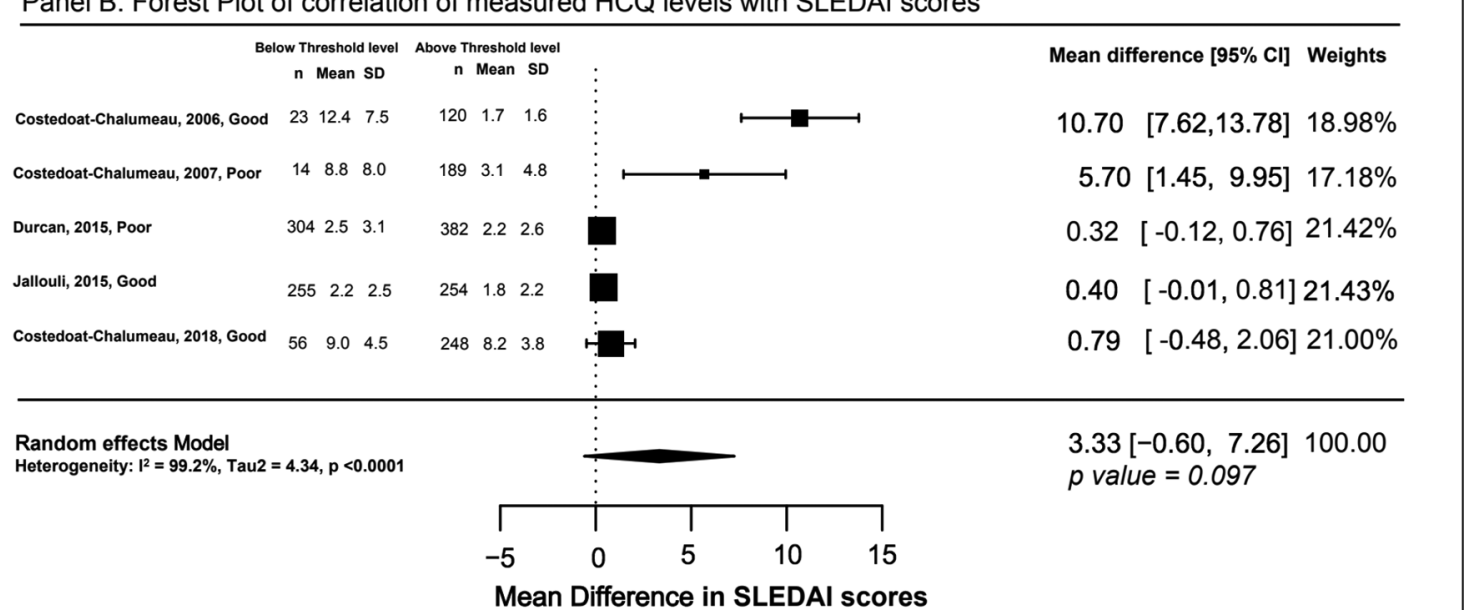

Abstract 221 Figure 1 Forest plot showing correlation of HCQ blood levels with A) Assessed Adherence B) SLEDAI scores

SLEDAI scores but due to the limited number of studies and heterogeneity we did not find significant correlation. Findings support the clinical utility of measuring HCQ levels in SLE patients. Future larger studies should investigate standards for HCQ level interpretation and target levels needed to minimize SLEDAI scores.

Funding Source(s): n/a

\section{CIRCULATING PENTRAXIN3-SPECIFIC B CELLS ARE DECREASED IN LUPUS NEPHRITIS}

${ }^{1}$ Mariele Gatto*, ${ }^{2}$ Annika Wiedemann, ${ }^{2}$ Nadja Nomovi, ${ }^{2}$ Karin Reiter, ${ }^{3}$ Eva Schrezenmeier, ${ }^{2}$ Thomas Rose, ${ }^{2}$ Franziska Szelinski, ${ }^{4}$ Andreia Lino, ${ }^{5}$ Sonia Valentino, ${ }^{6}$ Anna Ghirardello, ${ }^{7}$ Thomas Dörner, ${ }^{1}$ Andrea Doria. ${ }^{1}$ University of Padova, Department of Medicine, Division of Rheumatology; ${ }^{2}$ Department Medicine/Rheumatology and Clinical Immunology Charite' Universitätsmedizin Berlin, Germany; ${ }^{3}$ Department of Medicine/Nephrology and Medical Intensive Care Charité - Universitätsmedizin Berlin, Charitéplatz 1, 10117 Berlin; ${ }^{4}$ Deutsches Rheuma-Forschungszentrum, A Leibniz Institute, Berlin, Germany; ${ }^{5}$ Humanitas Clinical and Research Center, Milan, Italy; ${ }^{6}$ Unit of Rheumatology, Department of Medicine, University of Padova, Padova, Italy; ${ }^{7}$ Charite Universitätsmedizin Berlin and Deutsches Rheuma-Forschungszentrum (DRFZ)

10.1136/lupus-2019-Ism.222
Background Pentraxin3 (PTX3) is overexpressed in kidneys of patients developing lupus nephritis (LN). Active LN is associated with reduced anti-PTX3 antibodies. However, abnormalities of B cell differentiation against PTX3 have not been characterized in systemic lupus erythematosus (SLE).

The aim of our study is to characterize PTX3-specific $($ PTX3 +$)$ B cells in peripheral blood of SLE patients with or without LN and healthy donors (HD).

Methods SLE patients without LN, biopsy-proven LN and matched $\mathrm{HD}$ were analyzed. Active $\mathrm{LN}$ was defined as proteinuria $>0.5 \mathrm{~g} /$ day or $\mathrm{CrCl}<60 \mathrm{ml} / \mathrm{min} / 1.73 \mathrm{~m}^{2}$ with active urinary sediment. Peripheral B cells were analyzed for direct PTX3 binding by flow cytometry using PTX3 labeled with cyanine 5 (Cy5) and phycoerythrin (PE).

Results Initially, a flow cytometry based assay to identify PTX3 + B cells was developed by demonstrating simultaneous binding of PTX3-Cy5 and PTX3-PE. Specificity of B cells was validated by blocking experiments using unlabeled PTX3 (figure 1). We could identify circulating PTX $3+\mathrm{B}$ cells in HD and patients. Notably, LN patients showed a significantly diminished number of PTX3 +B cells (SLE vs. LN p=0.033; HD vs. LN $p=0.008$ ). This decrease was identified in naïve and memory $B$ cell compartments (naïve: SLE vs. LN 